\title{
Performance ANalysis of IMPROVEDAUTONOMOUS POWER CONTROL MAC PROTOCOL (IAPCMP) FOR MANETS
}

\author{
Sohan Kumar Yadav $^{1}$ and D. K. Lobiyal ${ }^{2}$ \\ ${ }^{1,2}$ School of Computer \& Systems Sciences, Jawaharlal Nehru University, New Delhi-67, \\ India

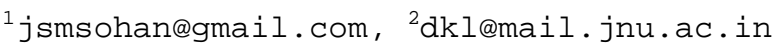

\begin{abstract}
Power Control in Mobile Ad Hoc networks is a critical issue, since nodes are powered by batteries. The main idea of power control schemes is to use different power levels for RTS/CTS and DATA/ACK. These schemes may degrade network throughput and reduce energy efficiency of the network. In this paper we have evaluated the performance of Improved Autonomous Power Control MAC Protocol (IAPCMP), that allows nodes to dynamically adjust power levels for transmission of DATA/ACK according to the distance between the transmitter and its neighbors.In IAPCMP power level for transmission of RTS/CTS is also adjustable. This also used maximum power level for transmitting DATA/ACK periodically to make neighboring nodes aware about ongoing transmission. The performance of IAPCMP is evaluated through the metrics namely, packet delivery ratio and rate of energy efficiency.The simulation results show significant improvement in protocol.
\end{abstract}

\section{KEYWORDS}

IEEE 802.11, Power Control, Energy Saving, Medium Access Control, Ad Hoc Network

\section{INTRODUCTION}

Mobile Ad Hoc Networks are multi-hop in nature and nodes are operated in distributed manner without any centralized infrastructure. The topology of the network is dynamically changes due to the nodes mobility. The nodes may join and leave the network frequently.The interests in such networks are from their ability to provide temporary and instant wireless networking solutions, where cellular infrastructures are not easily possible to establish $[1,13,15]$.

The Authors [2, 3, 6, 10] have given various power control schemes, out of these some are discussed here.In these schemes the power saving can be done in various ways.One way is to make use of some power saving mechanism, which let a node stay in a sleep state by powering off its wireless network interface, when no communication is required. Another alternative way is to use power control mechanism, which can adjust transmitting power level to save energy.

In [4,14], Authors are interested in controlling transmit power level to reduce energy consumption and to increase end-to-end delivery. It should be noted that in Mobile Ad Hoc Networks transmitting power level control is important for two reasons. One reason is it affects the traffic carrying capacity. And other is it also affects the spatial reuse i.e. simultaneous transmission.Since Mobile Ad Hoc Networks are dynamic in nature, therefore, to design an

DOI : 10.5121/ijans.2012.2402 
efficient power control protocol for MAC is a challenging task. Various energy efficient MAC schemes have been proposed for power control in the literature. However, very few schemes conserve energy without degrading the performance of the networks. But most of the schemes conserve energy by degrading throughput, delay or other metrics $[11,12]$.

We define some terms to make clear understanding on the protocol. So, here given the basic definitions of transmission range, carrier sensing range, and carrier sensing zone [16,17].

Transmission Range- Nodes in transmission range are able to decode data packets correctly. When two nodes transmit simultaneously, there are RTS/DATA transmission range of the sender and CTS/ACK transmission range of the receivers.

Carrier Sensing Range- Nodes in a carrier sensing range can sense the sender's transmission. This is usually larger than the transmission range. It is generally two times larger than the transmission range.

Carrier Sensing Zone - The area in which nodes can sense the incoming signals but cannot decode it correctly, is called carrier sensing zone.

In MAC protocols collision may occurs, to explain collision we explain the term hidden terminal and collision area as follows.

Collision Area- It is area in which hidden terminals are present. In the collision area, nodes are neither in RTS/CTS transmission range, nor in DATA/ACK transmission range. But these may inside the carrier sensing zone of the RTS or CTS. These may at beginning know the ongoing transmission, but lose sense later. Some nodes in the collision areas may sense the transmission for a while but they lost it eventually because of the power decrease (as happen in BASIC) or because of the asymmetry of the link. As shown in figure 1. The shadow parts in figure 1 denote the collision area, which are called left and right collision area. The transmitting nodes (such as a node A) in the left collision area will possibly affect the DATA reception in node D, while the node (as node F) in the right collision area may interfere with the ACK received by node $\mathrm{C}$.

Hidden Terminal- A node is in the receiver's carrier sensing zone, but is not in sender's carrier sensing zone or transmission range, and it may cause a collision to the reception of a DATA packet at the receiver. As shown in figure 1 , node $\mathrm{F}$ is the hidden terminal when node $\mathrm{C}$ transmits RTS to node D. similarly, node A is the hidden terminal when node D transmits CTS to node C.

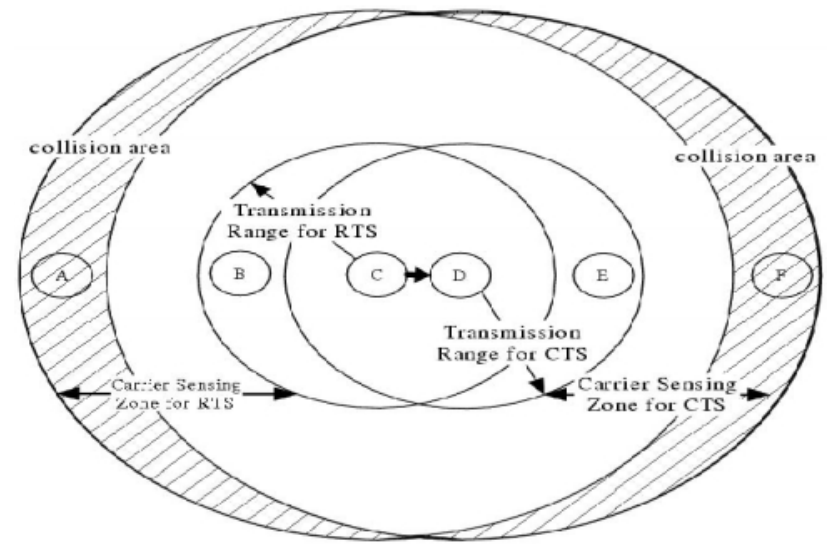

Figure 1 Hidden Terminal and Collision Area [17] 
In this paper we have evaluated the performances of Improved Autonomous Power Control MAC Protocol (IAPCMP) that enhance energy efficiency and also improve the end-to-end delay.

The rest of this paper is organized as follows. Section 2 presents the work related to the area. Section 3 deals a brief introduction of IAPCMP and its analysis with the other metrics. In section 4 , we discussed simulation and performance analysis of IAPCMP. Section 5 concludes the works and gave the possible future extension.

\section{RELATED WORK}

The IEEE 802.11 standards proposed for wireless LANs have been widely applied to ad hoc wireless networks. The IEEE 802.11 specifies the parameters for both physical (PHY) and medium access control (MAC) layer of a network. The MAC layer is the host for a set of protocols, which are responsible for regulating the use of a shared medium. IEEE 802.11 is the mostly used MAC protocol [5,7].

\subsection{IEEE 802.11 MAC Protocol}

IEEE 802.11 MAC protocol is a widely adopted one, in which the header of RTS (Request to Send), CTS (Clear to Send), and DATA include time duration to indicate when ACK can be received, that is, if a node can decode RTS, CTS or DATA correctly, it will know the on-going transmission and can avoid collision at the very first stage.In this scheme, RTS/CTS are send by using maximum transmit power level, and DATA/ACK are also send by maximum transmit power level as specified in CTS, RTS respectively. Since here we use same power level for RTS/CTS and DATA/ACK transmission, so it consumes more energy and also reduces the spatial reuse $[6,8]$.

\subsection{The BASIC Power Control MAC Protocol}

The Basic Power Control MAC protocol uses different transmitting power levels in the handshake RTS/CTS frames and DAT/ACK frames [4, 6]. When a node transmits the RTS/CTS, maximum power is used to make all other nodes know the ongoing transmission; while a node transmits

DATA/ACK lower power should be used. Let $p_{\text {desired }}$ be the power level for transmitting DATA/ACK, and is given by

$$
p_{\text {desired }}=\frac{p_{\max }}{p_{r}} * R_{x t h r e s h} * \beta
$$

Where $R_{x t h r e s h}$ is the minimum signal strength necessary to receive the signal, which is determined by the physical characteristics of the node, and $\beta$ is a constant.This protocol may introduce more collisions. The more collisions means more retransmission, and then higher throughput will be achieved by higher energyconsumption. This scheme will not beneficial for us because we are interested in finding higher throughput in less energy consumption. However, if mobility is present, the low power level transmission will also cause more retransmission, and thus it will consume higher energy.

\subsection{Autonomous Power Control MAC Protocol}

This protocol can adjust the transmitting power level for DATA/ACK and RTS/CTS packets both with respect to the current network situation [6]. This process of adjusting transmitting power 
level may result in different power levels used by different transmitters in the network. This may cause excessive collision, and it is likely to impossible to calculate the optimal transmitting power level according to the global network condition dynamically. A wireless channel uses shared medium. Therefore excessive high power level causes interference. This reduces traffic carrying capacity, and battery life of the network. However, if the mobility is present this protocol degrades the network efficiency.

\subsection{Improved Autonomous Power Control MAC Protocol}

This protocol can adjust the transmitting power level for RTS/CTS and DATA/ACK packets as done by Autonomous Power Control MAC protocol. Here Author [6,18], uses maximum power level $p_{\max }$ for data transmission periodically instead of $p_{\frac{\text { data }}{a c k}}$ when first data collision occurs.

The performance of this protocol evaluated in terms of throughput in mobile and static scenario. Therefore,throughput is not sufficient to evaluate the performance of any protocol. So, we have extends our evaluation of this protocol in terms of end-to-end delay and energy consumption.

\section{Performance Analysis of IAPCMP}

In this section, we discuss the Improved APCMP for MANETs [18].This protocol (IAMPCP) is considered as improved version of Autonomous power control MAC protocol [6].

In this work we perform the network analysis of IAPCMP in terms of delay ratio and rate of energy efficiency. More the rate of energy efficiency means the protocol is more efficient.The IAPCM protocol can adjust the transmitting power level for DATA/ACK packets as well as RTS/CTS packets according to the network situation in order to reduce the energy consumption. In IAPCMP transmitting power level for a local node group is adjusted in two ways. The first is to follow an appropriate power level for transmitting DATA/ACK, depending on the average distance from the transmitter to all current neighbors. The other is to adjust the power level for transmitting next RTS/CTS to a value proportional to the DATA/ACK power level. Therefore, the energy consumption can be lowered by collision avoidance.

In a MAC protocol, we know that the distance can be estimated by using the transmitting power level at a transmitter and the actual received signal power level at a receiver. Therefore, a bidirectional links between transmitter and receiver can be ensured, as long as the transmitter/receiver transmits packets using some suitable power level, at which the receiver/transmitter can receive the same signal power level. Using this estimated distance information, the required power level can be calculated. Based on this consideration, in IAPCMP, transmitting power for sending RTS/CTS packets is adjusted to a level just slightly higher than that is required for transmitting DATA/ACK packets. We also increase the transmitting power level for transmitting DATA/ACK periodically to overcome the hidden terminal problem. The time period to increase the transmit power is considered less than the SIFS time. Further, to ensure connectivity of the network, we increase transmitting power level gradually, if it is too low to reach any other node $\mathrm{n}$ the network.

\subsection{Calculating for Transmitting Power Levelin IAPCMP}

In this protocolwe give the process by which the transmission power can be calculated which is similar to the authors [6] idea. The only difference in my calculation is that we are taking $k=4$. In this the distance information is used to determine the transmitting power level. It is also 
assumed that the noise level at a receiver is less than the signal level. The signal attenuation is given by

$d=\sqrt[4]{\frac{p_{r t s}}{p_{r e c}}} * \varepsilon$

Where, $p_{r t s} / c t s$ is transmitting power level for RTS/CTS packets, $p_{r e c}$ is the received signal power level, and $\varepsilon$ is a constant dependent on the antenna gain, system loss and wavelength. Here we set it to 1 .

For a given transmitter, suppose that there are $(k-1)$ mobile nodes as its neighboring nodes. Let us consider a transmitter wants to send a data packet to another mobile node, say $k^{\text {th }}$ node. It firstly calculates the distance from the transmitter to the $k^{\text {th }}$ node. The average estimated distance from the transmitter to the $k^{\text {th }}$ mobile nodes, is given by

$d^{\prime}=\frac{1}{k} \sum_{i=1}^{k} d_{i}$

Where $d_{i}$ is the estimated distance from the transmitter to the $i^{\text {th }}$ neighboring node.

The power level for transmitting DATA/ACK packets from transmitter to the $k^{\text {th }}$ node is given by

$$
p_{\frac{d a t a}{a c k}}=d^{14} * R_{x t h r e s h}
$$

The estimated distance between transmitter and the $k^{\text {th }}$ node is represented by $d_{k}$, and $d^{\prime}$ is the average of estimated distance of all $k$ neighboring nodes. If $d_{k}>d^{\prime}$, transmitted via its neighbors which transmitter cancels the transmission due to insufficient transmission power level. The data packet has to be transmitted via its neighbors which are close to it.

The transmitting power level for the next RTS/CTS is calculated as follows

$$
p_{\frac{r t s}{c t s}}^{\prime}=p_{\frac{d a t a}{a c k}} * \beta
$$

Where $\beta$ is a parameter related to the network situation and here it is $\beta>1$.

\subsection{Transmission cycle for packets in IAPCMP}

The transmission cycle of IAPCMP consists of five steps, which are as follows [18]:

Firstly, a transmitter sets the value of $p_{\frac{r t s}{c t s}}\left(p_{r t s T}\right)$ and $p_{a c k R}$, which is stored in the routing table and in the RTS packet. Then the transmitter sends RTS packet using power level $p_{\frac{r t s}{c t s}}$. 
The receiver receives RTS packet by power level $p_{r e c}$ and obtains the information about $p_{\frac{r t s}{c t s}}$, $p_{a c k R}$. With this information, it calculate the desired power level for DATA packets transmission as follows

$$
p_{\text {dataT }}=\frac{p_{r t s T}}{p_{\text {rec }}} * R_{x t h r e s h}
$$

The receiver estimates distance from the current transmitter by using $p_{a c k R}$ with the estimated distance, New power level $p_{\frac{d a t a}{a c k}}$ and $p_{\frac{r t s}{c t s}}^{\prime}$ for the receiver is calculated from the equation 4 and 5. The new value of $p_{\frac{r t s}{c t s}}^{\prime}$ is set as the new RTS/CTS for receiver. The receiver sends the values of $p_{\frac{r t s}{c t s}}\left(p_{c t s R}\right)$ and $p_{\text {dataT }}$ in the CTS packet to the transmitter with the new power level $p_{\frac{r t s}{c t s}}$.

Transmitting node receives the CTS packet by $p_{\text {rec }}$ and obtains the information carried by it. Using this information the desirable power level $p_{a c k R}$ for the receiver to transmit ACK packet is given by

$p_{a c k R}=\frac{p_{c t s R}}{p_{\text {rec }}} * R_{x t h r e s h}$

Then, transmitter calculates and saves the estimated distance to the current receiver $d_{r}$. The average distance $d^{\prime}$ to all the neighbors is calculated. Then it can calculate- $p_{\frac{d a t a}{a c k}}, p_{\frac{r t s}{c t s}}^{\prime}$, and $p_{\frac{r t s}{c t s}}^{\prime}$ as new transmission power for RTS/CTS. For data transmission it use $p_{\frac{\text { data }}{a c k}}$.

If the transmitting node does not receive CTS packet after a timeout, it increases the power level for transmitting RTS/CTS to the previous used value and send RTS again.

We use the maximum power level $p_{\max }$ for data transmission periodically instead of $p_{\frac{\text { data }}{a c k}}$ whenever first data collision occurs. The time for periodically use of $p_{\max }$ is set to less than SIFS.After receiving the DATA packet, receiver send back ACK packet using the power level $p_{\frac{d_{\text {data }}}{a c k}}$. If the transmitter receives ACK before the timeout, transmission cycle is completed successfully. Otherwise, the transmitter transmits the DATA packet again in a same way up to the maximum retransmission limit.

\section{PerformanceEvaluation}

In this section, we evaluated the performance of the IAPCMP [18] with network simulator GloMoSim [9] and compared with other power control MAC protocols.

\subsection{Simulation Environment}

We have considered varying node density i.e. $4,8,12$, and 16 with and without mobility. Further the other parameters used in simulation are listed in table 1. The career sensing range is $550 \mathrm{~m}$. and the highest power level at $24.5 \mathrm{dBm}$. 
International Journal on AdHoc Networking Systems (IJANS) Vol. 2, No. 4, October 2012

Table 1. Simulator Parameters

\begin{tabular}{|l|l|}
\hline Simulation Time & 1000 second \\
\hline Network Area & $500 \times 500$ \\
\hline Radio Transmission Range & $250 \mathrm{~m}$ \\
\hline Propagation model & two-ray path loss \\
\hline Bandwidth & $2 \mathrm{Mbps}$ \\
\hline Node Placement & Random \\
\hline Mobility-Wp-pause & $0.1 \mathrm{Millisecond}$ \\
\hline Mobility-Wp-Min-Speed & $0 \mathrm{~m} / \mathrm{s}$ \\
\hline Mobility-Wp-Max-Speed & $10 \mathrm{~m} / \mathrm{s}$ \\
\hline Noise Figure & 4 \\
\hline Radio-Tx-Power & $24.5 \mathrm{dBm}$ \\
\hline Radio-Antenna-Gain & $0.0 \mathrm{dBm}$ \\
\hline Radio-Rx-Sensitivity & $-71.42 \mathrm{dBm}$ \\
\hline Mobility Model & Random Way Point \\
\hline Routing & LAR1 \\
\hline Promiscuous-Mode & No \\
\hline
\end{tabular}

\subsection{Simulation Results}

We used following metrics to evaluate the performance of the IAPCMP, APCMP, BPCMP, and IEEE 802.11 protocols under both the mobile and static environment. In mobile condition the maximum speed is considered $10 \mathrm{~m} / \mathrm{s}$.

Delivery Ratio: It is the total number of data packets successfully delivered out of the total number of data packets sent. This shows an external measure of connectivity performance.

\section{Delivery Ratio $=$ Total number of packets deliver $/$ Total number of packets sent}

Rate of Energy Efficiency: It is the number of data bits delivered/joule energy consumed. Higher the rate of energy efficiency means protocol more efficient.

Rate of Energy Efficiency $=$ total number of data bits / total energy consumed (joule)

Figure 2 shows the delivery ratio for different network size of $4,8,12$, and 16 nodes in a static situation. Therefore, from results we conclude that the delivery ratio of IAPCMP is comparatively better than other protocols i.e. BPCMP, APCMP, etc. 
International Journal on AdHoc Networking Systems (IJANS) Vol. 2, No. 4, October 2012

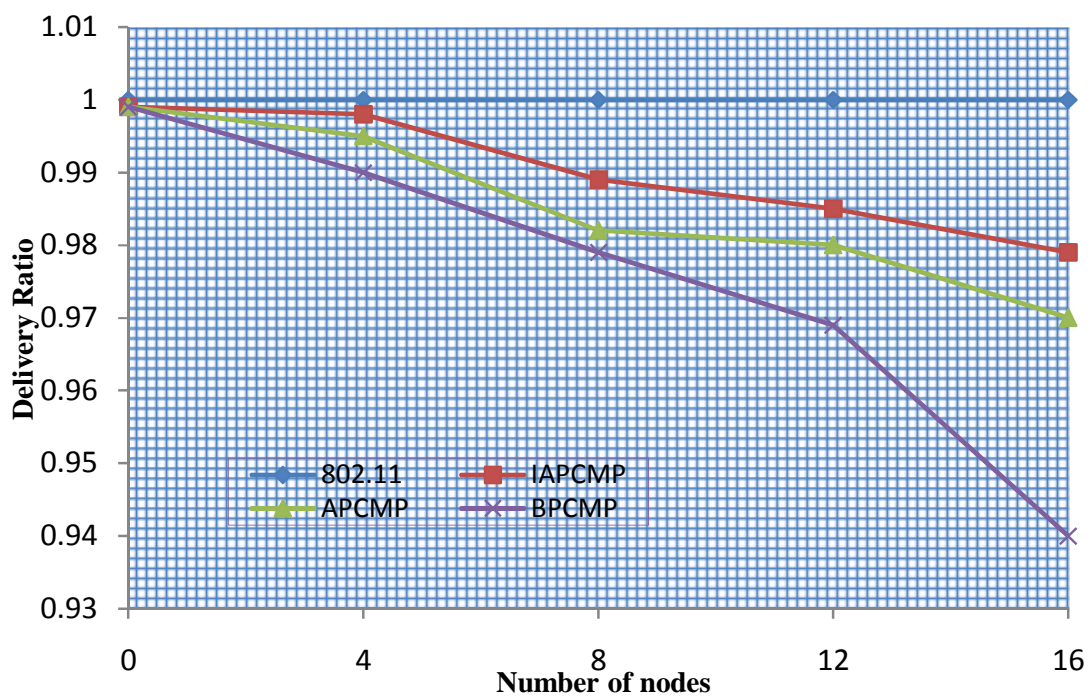

Figure 2. Comparison of Delivery Ratio in Static Situation

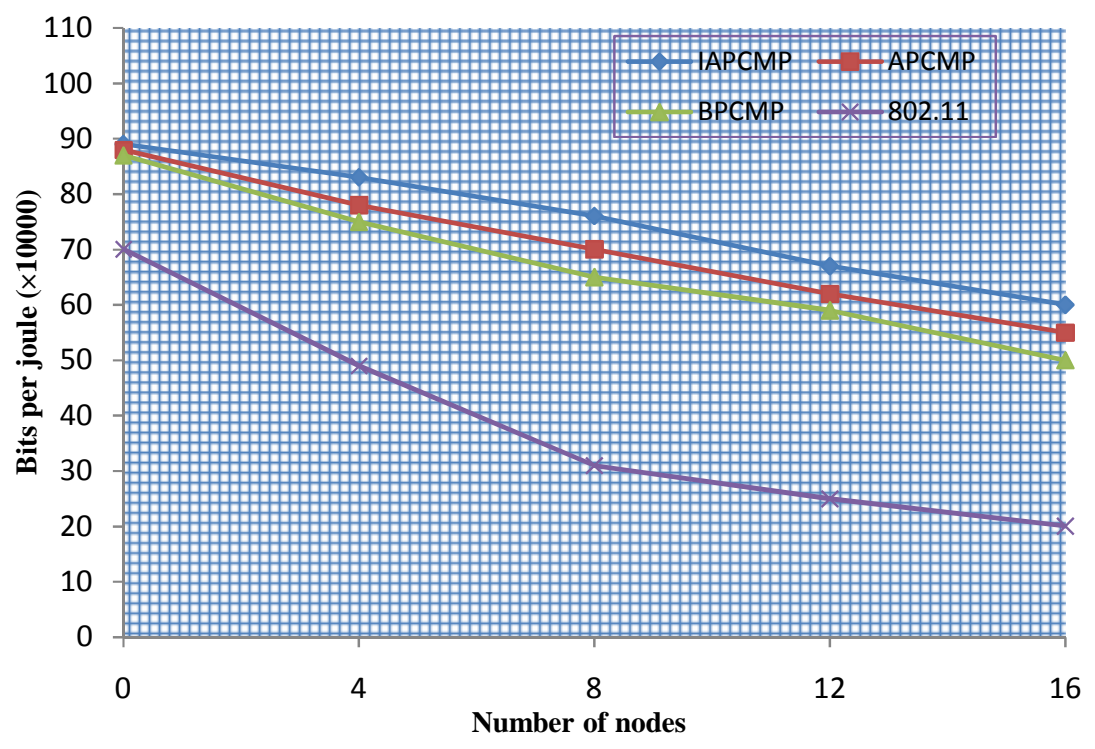

Figure 3.Comparison of Energy Efficiency in Static Situation 
International Journal on AdHoc Networking Systems (IJANS) Vol. 2, No. 4, October 2012

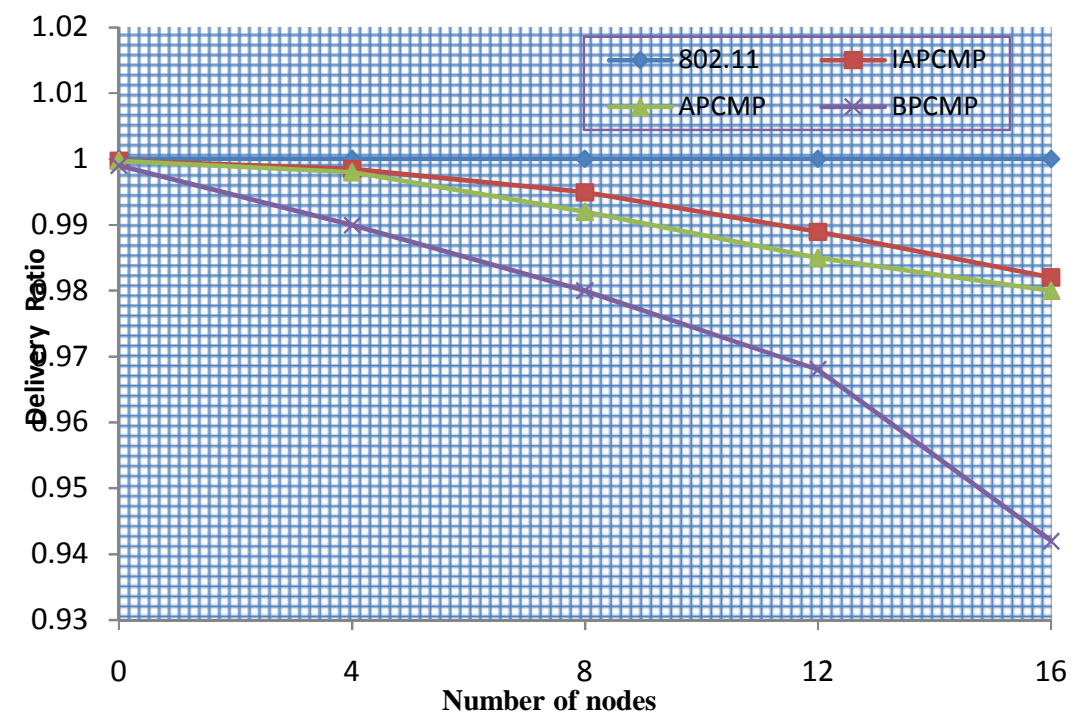

Figure 4. Comparison of Delivery Ratio in Mobile Condition(max speed $10 \mathrm{~m} / \mathrm{s}$ )

Figure 3 shows the rate of energy efficiency for different network size of 4, 8, 12, 16 nodes in static situation. Therefore, from the figure 3 it is clear that IMPCMP is more energy efficient than the BPCMP, APCMP, IEEE 802.11.

Figure 4 shows the delivery ratio of network in mobile environment with maximum moving speed of a node is $10 \mathrm{~m} / \mathrm{s}$. Figure 4 tells that the IAPCMP performs better than BPCMP, and other protocols.

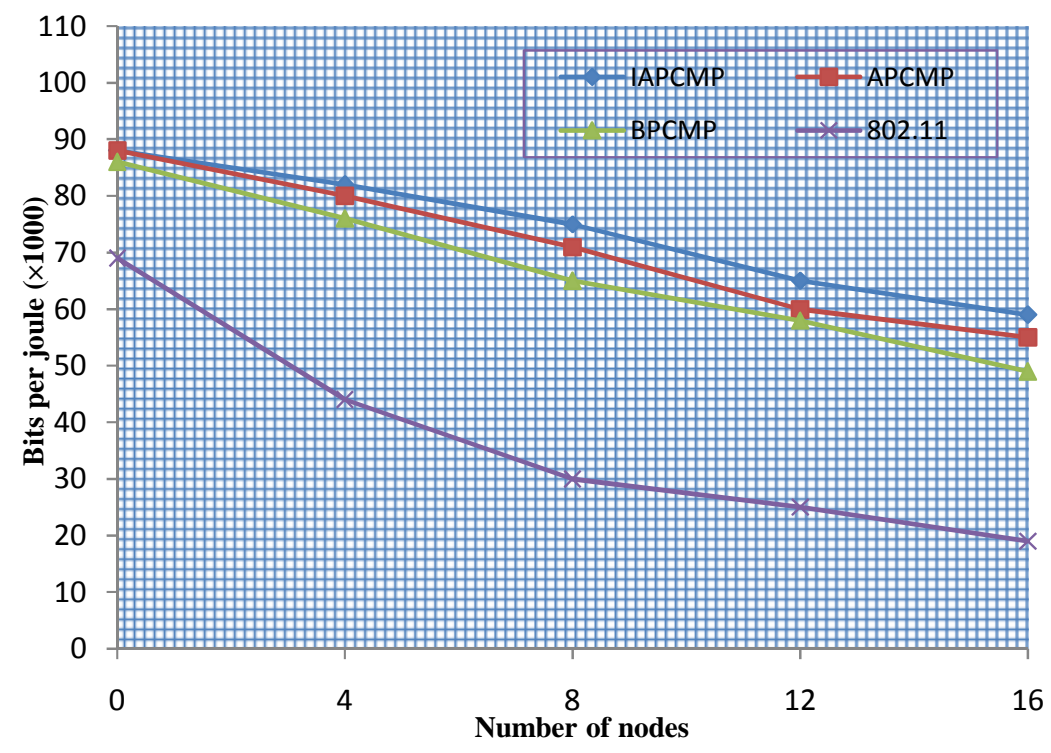

Figure 5.Comparison of Energy Efficiency in Mobile Condition (max speed $10 \mathrm{~m} / \mathrm{s}$ )

Figure 5 clearly shows that energy efficiency of proposed protocol in moble situation with maximun moving speed of node $(10 \mathrm{~m} / \mathrm{s})$ is better in comparision to APCMP, BPCMP, IEEE 802.11 . 


\section{Conclusions}

Mobile Ad Hoc Networks are powered by batteries and there is no centralized administration. Therefore, to reduce overall energy consumption is vital for providing quality of service in mobile ad hoc networks. Thus, to increase the network performance, improved autonomous power control MAC protocolfor mobile ad hoc networks can adjust transmitting power level automatically as well as periodically according to the network situation. It reduces collision except first one. Therefore, this protocol saves energy without degrading data delivery ratio.It also increases rate of energy efficiency. From the simulation results obtained, it is clear that IAPCMP has better results than BPCMP, APCMP, and IEEE 802.11in terms of metrics end-toend delay and energy efficiency. It also serves the purpose of energy conservation through power control, and decreases collision rate. IAPCMP does not degrade the network quality as generally other protocols do while conserving energy. Therefore, from the simulation results it is shown that IAPCMP yields significant improvement in power savingand delivery ratio of the network.

\section{ACKNOWLEDGEMENTS}

The work presented in this paper was supported by Council of Scientific \& Industrial Research (Human Resource Development Group), New Delhi, India.

\section{REFERENCES}

\section{Authors}

[1] Murthy, C. S. Ram\&Manoj, B.S.,(2005) “Ad Hoc Networks: Architectures and Protocols”, Second Edition, Pearson Education.

[2] Chang, C.Y. \& Chang, H.R., (2007) "Power Control and fairness MAC mechanisms for 802.11 WLWNs", Computer Communications 30, pp1527-1537.

[3] Jung, E.S. \&Vaidya, N. H., (2005) "A Power Control MAC Protocol for Ad Hoc Networks",ACM/Kluwer journal on wireless networks (WINET), Vol. 11, pp55-56.

[4] Jung, E.S. \&Vaidya, N.,(2002) "An Energy Efficient MAC Protocol for wireless LANs", Inproceedings of INFOCOM.

[5] F. Ye, S. Yi \& B. Sikda., (2003) "Improving Spatial reuse of IEEE 802.11 based Ad Hoc Networks", Proc. IEEE GLOBECOM.

[6] Chen, H.H., Fan, Z. \&Li, J., (2006) "Autonomous Power Control for MAC Protocol for Mobile Ad Hoc Networks",Hindwani Publishing Corporation, EURUSIP Journals on Wireless Communication and Networking, Vol. 2006, Article ID 36040, pp 01-10.

[7] Gomez, J., Campbell, A.T., Naghshineh, M.\&Bisdikian, C., (2001)“Conserving Transmission Power in Wireless Ad Hoc Networks", In ICNP 01.

[8] Monks, J.P., Ebert, J.P., Wolisz, A. \&Hwu, W.M.W., (2001)“A Study of the Energy Saving and Capacity Improvement Potential of Power Control in Multi-hop Wireless Networks”, In proceeding of 26th Annual IEEE Conference on Local Computer Networks (LCN) and Workshop on Wireless Local Networks (WLN), Tampa (FL), USA.

[9] Nuevo, J., (2004) A Comprehensible GloMoSim Tutorial.

[10] Xu, K., Gerla, M. \&Bae, S., (2002)"How Effective is the IEEE 802.11 RTS/CTS Handshake in Ad Hoc Networks?", In Proceeding GLOBECOM 2002, Taipei.

[11] Qin, L. \&Kunz, T., (2004)“Survey on Mobile Ad Hoc Network Routing Protocols and Cross-layer Design”, Carleton University, Systems and Computer Engineering, In Technical Report SCE,pp0414.

[12] Krunz, M. \& Muqattash, A., (2004)“Transmission Power Control in Wireless Ad Hoc Networks; Challenges, solutions, and open issues", The University of Arizona Sung-Ju Lee, Hewletl-Packard Laboratories, IEEE Network. 
International Journal on AdHoc Networking Systems (IJANS) Vol. 2, No. 4, October 2012

[13] Gupta, P. \&Kumar, P.R., (2000)“The Capacity of Wireless Networks", IEEE Transaction on information theory, vol. 46 no. 2, pp388-404.

[14] Agarwal, S., Krishnamurthy, S., Katz, R.H. \&Dao, S.K. (2001)“Distributed Power Control in Ad-hoc wireless networks", In PIMRC01.

[15] Kawadin, V. \&Kumar, P.R., (2003)“Power Control and Clustering in Ad Hoc Networks”, Proc. Of IEEE. INFOCOM, pp459-469.

[16] Varvariyos, Emmanouel A., Vasileios, G. \& Nikolaos, K. (2009) “The slow start power control MAC protocol for mobile ad hoc networks and its performance analysis", Ad Hoc Networks 7, pp11361149.

[17] Yan, Hairong., Li, J., Sun, G., Chen \& Haiaso-Hwa (2006) "An optimistic power control MAC protocol for mobile Ad Hoc Networks”, IEE ICC Proceedings, pp3615-3620.

[18] Yadav, SohanKumar\&Lobiyal, D.K. (2012) "Improved Autonomous Power Control MAC Protocol for MANETs", In proceedings of Second International Conference on Advances in Computing and Information Technology (ACITY), 13-15thJuly 2012 (Springer), pp 553-562.

\section{Authors}

\section{Sohan Kumar Yadav}

Received his Master of Technology in Computer Science from Jawaharlal Nehru University, India in2010. Presently, he is aresearch scholar in Computer Science from the School of Computer and Systems Sciences, Jawaharlal Nehru University, India. His areas of research interest are Mobile Ad hoc Networks, operation research.

\section{K. Lobiyal}

Received his Bachelor of Technology in Computer Science from Lucknow University, India, in 1988 and his Master of Technology and $\mathrm{PhD}$ both in Computer Science from Jawaharlal Nehru University, New Delhi, India, 1991 and 1996, respectively. Presently, he is an Associate Professor in the School of Computer and Systems Sciences, Jawaharlal Nehru University, India. His areas of research interest are Mobile Ad hoc Networks, Vehicular Ad Hoc Networks, Wireless Sensor Network and Video on Demand.

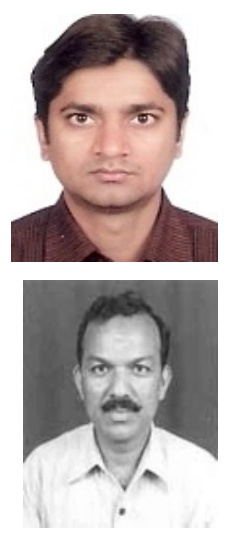

